\title{
Influences of Short-Wave Truncations to Spectral Energy Budget in Hall MHD Turbulence
}

\author{
Hideaki MIURA \\ National Institute for Fusion Science, Gifu 509-5292, Japan
}

(Received 11 December 2009 / Accepted 17 February 2010)

\begin{abstract}
The effect of a sharp short-wave truncation on Hall magnetohydrodynamic (MHD) turbulence is studied numerically to obtain basic information for constructing sub-grid-scale models of the Hall MHD equations. Hall MHD turbulence is found to be less sensitive to truncation than MHD turbulence, because the Hall term suppresses energy transfer in the magnetic field at relatively low wave numbers.
\end{abstract}

(c) 2010 The Japan Society of Plasma Science and Nuclear Fusion Research

Keywords: Hall MHD, isotropic turbulence, truncation

DOI: $10.1585 / \mathrm{pfr} .5 . S 2059$

\section{Introduction}

One-fluid magnetohydrodynamic (MHD) equations are a good platform for studying the macroscopic behaviors of fusion plasmas. However, as the numerical resolutions of MHD simulations increase, the scales resolved in the simulations are going to overlap with those of parameters neglected in the MHD approximation, such as the ion skin depth and the finite Larmor radius. For example, full-three-dimension (3D) MHD simulations in the Large Helical Device (LHD) resolve the evolutions of moderatewave-number ballooning modes, the wave length of which is comparable to the ion skin depth [1]. Thus for simulations at finer resolutions, we expect the emergence of turbulence due to non-MHD effects. However, simulating macroscopic instabilities and micro-turbulence simultaneously in a realistic torus geometry is not easy. To reconcile the two kinds of computations, it may be preferable to model sub-grid scale effects and perform so-called large eddy simulations (LES) of an extended-MHD system.

While the LES approach is quite attractive for inexpensive computations, sub-grid-scale models for extendedMHD equations are not well established. In this paper, to obtain basic information for sub-grid-scale modelings of fusion plasmas, the effect of sharp low-pass filtering (Fourier wave-number truncation) on Hall MHD turbulence is studied by means of direct numerical simulations (DNSes) and truncated simulations. The Hall MHD equations are the among simplest extended-MHD equations. While one-fluid MHD turbulence (hereafter simply "MHD turbulence") has been studied extensively [2], studies on Hall MHD turbulence are now under way $[3,4]$. For simplicity, we assume that the the fluid velocity is incompressible. We also restrict ourselves to the study of homogeneous and isotropic turbulence without a background magnetic field. This paper is organized as follows. In section

author'se-mail: miura.hideaki@nifs.ac.jp
2, outlines of numerical simulations of the 3D Hall MHD equations and the MHD equations are presented. In section 3 , numerical results are reported. A summary is presented in section 4.

\section{Numerical Simulations}

DNSes and truncated simulations of decaying, homogeneous and isotropic turbulence in incompressible Hall MHD equations and in incompressible MHD simulations are described out in this section. The incompressible Hall MHD equations are given by

$$
\begin{aligned}
\partial_{t} V_{i}= & -\partial_{j}\left(V_{i} V_{j}-B_{i} B_{j}\right)-\partial_{i}\left(p+B_{j} B_{j} / 2\right) \\
& +v \partial_{j} \partial_{j} V_{i}, \\
\partial_{j} V_{j}= & 0, \\
\partial_{t} B_{i}= & \epsilon_{i m n} \partial_{m}\left[\epsilon_{n \alpha \beta}\left(V_{\alpha}-\epsilon j_{\alpha}\right) B_{\beta}-\eta j_{n}\right],
\end{aligned}
$$

where $V_{i}, B_{i}$, and $j_{i}=\epsilon_{i m n} \partial_{m} B_{n}$ are the $i$-th component of the velocity field vector $\boldsymbol{V}$, the magnetic field vector $\boldsymbol{B}$, and the current density vector $\boldsymbol{j}$, respectively. We also introduce the vorticity vector field $\omega$, the $i$-th component of which is given as $\omega_{i}=\epsilon_{i m n} \partial_{m} V_{n}$. The symbols $\epsilon_{i m n}, v$, and $\eta$ are Eddington's anti-symmetric tensor, the viscosity and the resistivity, respectively. We take the sum from 1 to 3 over a repeated subscript. The temporal and spatial derivatives are expressed as $\partial_{t}:=\partial / \partial t$ and $\partial_{i}:=\partial / \partial x_{i}$, respectively. The symbol $\epsilon$ is the Hall term, which comes from the ion skin depth. By setting $\epsilon=0$, we obtain the MHD equations.

In the DNSes, eqs.(1)-(3) are solved by the pseudospectral method and the Runge-Kutta-Gill scheme under the triple periodic boundary condition over a $(2 \pi)^{3}$ cube. The number of grid points is $N^{3}=512^{3}$. The aliasing errors in the pseudo-spectral computations are removed by the 2/3-rule. Because of this rule, the maximum wavenumber in the simulations is limited to $k_{\max }=170$.

Truncated simulations are performed to study the ef- 
fect of the low-pass filter on the two kinds of turbulence. A sharp low-pass filter with a cut-off wave-number $k=k_{c}$ is applied to eqs.(1)-(3). The resulting equations are

$$
\begin{aligned}
\partial_{t} \bar{V}_{i}= & -\partial_{j}\left(\bar{V}_{i} \bar{V}_{j}-\bar{B}_{i} \bar{B}_{j}\right)-\partial_{i}\left(\bar{p}+\bar{B}_{j} \bar{B}_{j} / 2\right) \\
& +v \partial_{j} \partial_{j} \bar{V}_{i}+\partial_{j} W_{i j}^{V}, \\
W_{i j}^{V}= & -\partial_{j}\left[\left(\overline{V_{i} V_{j}}-\bar{V}_{i} \bar{V}_{j}\right)-\left(\overline{B_{i} B_{j}}-\bar{B}_{i} \bar{B}_{j}\right)\right] \\
& -\partial_{i}\left(\overline{B_{j} B_{j}}-\bar{B}_{j} \bar{B}_{j}\right) / 2, \\
\partial_{j} \bar{V}_{j}= & 0, \\
\partial_{t} \bar{B}_{i}= & \epsilon_{i m n} \partial_{m}\left[\epsilon_{n \alpha \beta}\left(\bar{V}_{\alpha}-\epsilon j_{\alpha}\right) \bar{B}_{\beta}-\eta \bar{j}_{n}\right] \\
& +\partial_{j} W_{i j}^{M}, \\
W_{i j}^{M}= & \epsilon_{k m n}\left[\overline{\left(V_{m}-\epsilon j_{m}\right) B_{n}}-\left(\bar{V}_{m}-\epsilon \bar{j}_{m}\right) \bar{B}_{n}\right] .
\end{aligned}
$$

Here the symbol - represents a filtered quantity. To close the eqs.(4)-(7), we need to express $W_{i j}^{V}$ and $W_{i j}^{M}$ in terms of $\bar{V}_{i}, \bar{B}_{i}$, and $\bar{j}_{i}$. Typically, the equations are closed by adopting the eddy viscosity model $W_{i j}^{V}=v_{e d d y} \partial_{j} \bar{V}_{i}$ in an LES. However, it is not clear yet whether the eddy viscosity model works for Hall MHD turbulence. In fact, the eddy viscosity model may not work for simulations with a strong magnetic field, because the $\boldsymbol{j} \times \boldsymbol{B}$ term plays a significant role not only in the velocity equation but also in the induction equation. Here, we simply set $W_{i j}^{V}=W_{i j}^{M}=0$ and study the effect of the Fourier truncation on the plasma dynamics. Equations (4)-(7) are solved numerically by the same numerical techniques as those used for the DNSes. The cut-off wave-number is set to $k_{c}=32$.

We carry out the DNSes and the truncated simulations for the parameters $v=\eta=2 \times 10^{-3}$, for both the Hall MHD turbulence $(\epsilon=0.05)$ and the MHD turbulence $(\epsilon=$ $0)$. The initial conditions are given by the energy spectrum $E_{\alpha}(k) \propto k^{4} \exp \left(-2 k^{2} / k_{0}^{2}\right)\left(k_{0}=2\right.$ here $)$ and the random phases for both the velocity field and the magnetic field. Hereafter, the numerical data from the DNSes of the Hall MHD and MHD turbulence are referred to as Hall MHD (DNS) and MHD (DNS), respectively, and data from the truncated simulations of the two turbulence are referred to as Hall MHD (truncated) and MHD (truncated).

\section{Simulation Results}

Figure 1 (a) shows the time evolution of the kinetic energy, $E_{K}:=\left\langle V_{i} V_{i} / 2\right\rangle$, for the four simulations, MHD (DNS), MHD (truncated), Hall MHD (DNS) and Hall MHD (truncated). The brackets $\langle\cdot\rangle$ represent the volume average. The $E_{K}$ values of the truncated runs decay slower than those of the DNSes. The slower decays in the truncated runs are attributed to the truncation of high wavenumber coefficients because the energies are mostly dissipated at high wave-numbers. The effect of the truncation on $E_{K}$ is greater in MHD turbulence than in Hall MHD turbulence, since the peak of $E_{K}$ at $t \simeq 0.2$ in MHD (DNS)
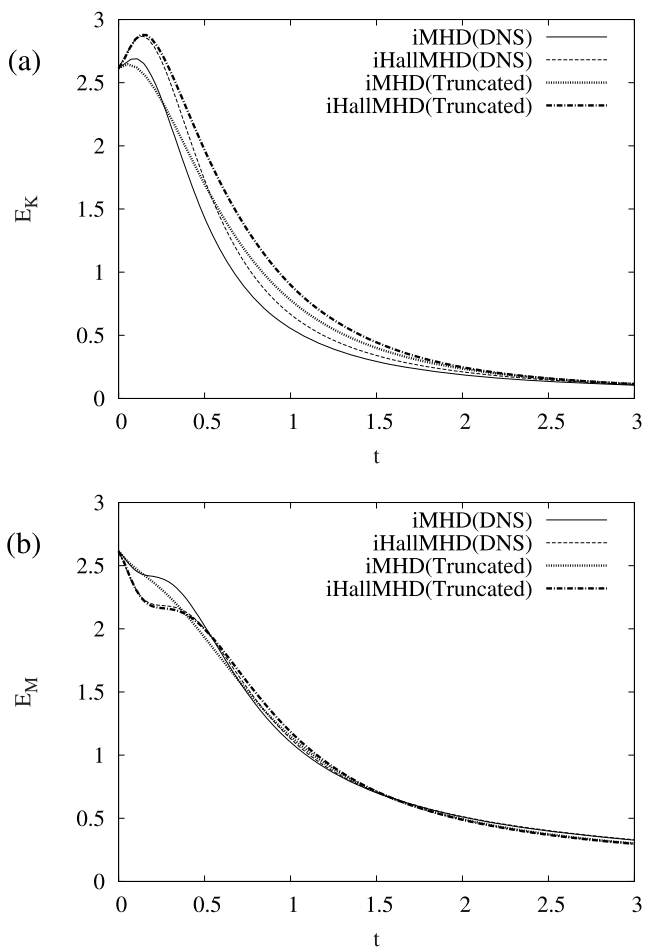

Fig. 1 Time evolutions of (a) $E_{K}$ and (b) $E_{M}$ in the MHD (DNS), MHD (truncated), Hall MHD (DNS) and Hall MHD (truncated) simulations.

becomes small in MHD (truncated). Note that the peak is formed through energy exchange between $E_{K}$ and the magnetic energy $E_{M}:=\left\langle B_{i} B_{i} / 2\right\rangle$. (We can see easily that the total energy $E_{K}+E_{M}$ decays monotonically in all the runs. See Ref. [5].) Figure 1 (b) shows the time evolution of $E_{M}$. In the two DNSes, $E_{M}$ decays initially and then experiences a short plateau at $t \lesssim 0.5$. This sequence is caused by the energy exchange with $E_{K}$. The initial decay of $E_{M}$ in Hall MHD (DNS) is steeper than that in MHD (DNS). Since the Hall term is the only difference between the Hall MHD and MHD equations, the difference in the decay rate is directly attributed to the Hall term. However, the Hall term is quadratic to $\boldsymbol{B}$ and essentially contributes only to energy transfer among scales of the magnetic field, not to energy exchange between $E_{K}$ and $E_{M}$. Therefore, the difference in the decay rates of $E_{M}$ should be understood as magnetic energy being first transferred quickly by the Hall term between scales, where energy transport from $E_{M}$ to $E_{K}$ works efficiently. In the evolution of $E_{M}$ in Hall MHD (truncated) in Fig. 1 (b), the two plots of Hall MHD (DNS) and Hall MHD (truncated) coincide well. This indicates that the energy transfer and subsequent energy exchange described above are insensitive to truncation. Unlike the case of Hall MHD turbulence, $E_{M}$ in MHD (truncated) does not show a plateau. This suggests that energy transfer in the magnetic field and/or energy exchange between $E_{K}$ and $E_{M}$ in MHD (truncated) are prevented by truncation, but those in Hall MHD (truncated) are not. Af- 
(a)

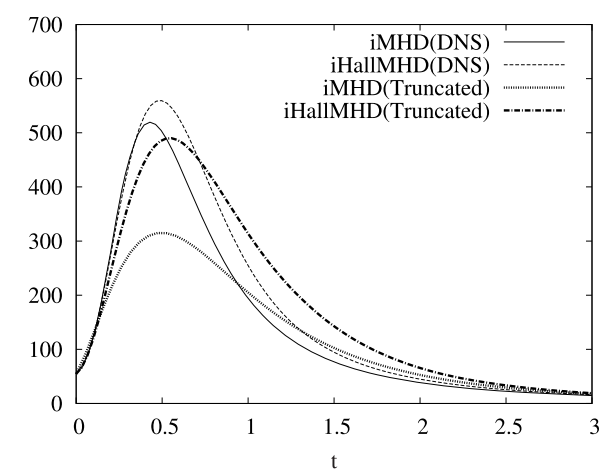

(b)

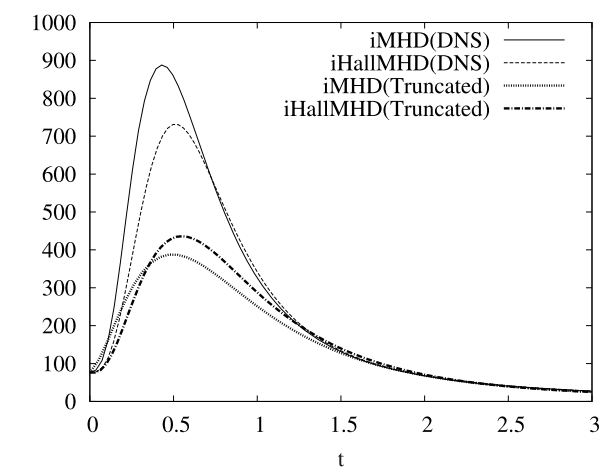

Fig. 2 Time evolutions of (a) $Q$ and (b) $J$ in the four simulations.

ter the plateau period, $E_{M}$ decays at almost the same rate in four runs, suggesting that truncation has little effect on the relaxation process.

Figure 2 (a) shows the time evolution of the enstrophy, $Q:=\left\langle\omega_{i} \omega_{i} / 2\right\rangle$. The peak value of $Q$ in Hall MHD (truncated) is about $87 \%$ of that in Hall MHD (DNS), whereas the value in MHD (truncated) is as small as about $60 \%$ of the value in MHD (DNS). Thus Hall MHD turbulence is less sensitive to truncation than MHD turbulence. The decay rate of $Q$ in Hall MHD (truncated) after the peak is smaller than that in Hall MHD (DNS). Figure 2 (b) shows the time evolution of the total current, $J:=\left\langle j_{i} j_{i}\right\rangle / 2$. The peak $J$ values in the truncated MHD and Hall MHD simulations are about $40 \%$ and $60 \%$, respectively, of those of the DNSes. Although Hall MHD turbulence is less sensitive than MHD turbulence in this aspect also, the reduction in $J$ appears very large. However, here we recall that effect of truncation on $E_{M}$ is not significant for Hall MHD turbulence. While $J$ is closely related to the magnetic energy diffusion at high wave-numbers and is reduced considerably by truncation, the reduction is not directly related to the behaviors of $E_{M}$. A consistent understanding may be that many of the physical mechanisms associated with the magnetic energy, such as the energy transfer and the subsequent energy exchange between $E_{M}$ and $E_{K}$, are dominated by large-scale motions. Since energy exchange in MHD turbulence is considerably suppressed by truncation as we have seen above, the insensitivity of Hall MHD turbulence to truncation should be closely related to energy transfer among scales in the magnetic field.
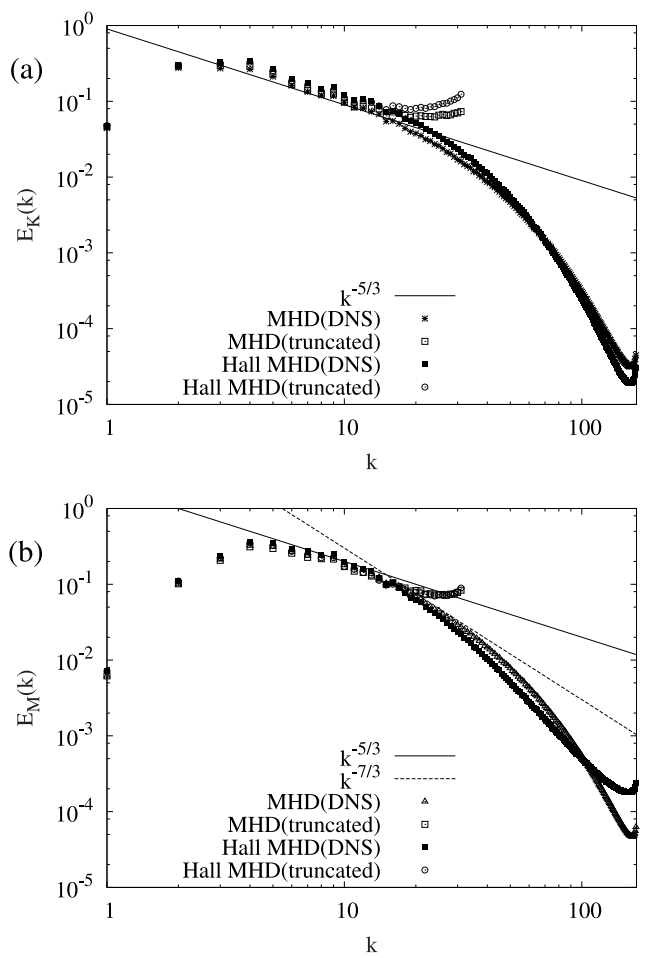

Fig. 3 Energy spectra of the kinetic energy $E_{K}(k)$ and magnetic energy $E_{M}(k)$.

Figure 3 (a) shows the kinetic energy spectrum, $E_{K}(k):=\sum_{[k]}\left|\widetilde{V}_{i}(k)\right|^{2}$, where $\sum_{[k]}$ denotes the shell average over $3 \mathrm{D}$ wave-number space, and $k:=\sqrt{k_{i} k_{i}}$. Figure 3 (b) shows the magnetic energy spectrum, $E_{M}(k):=$ $\sum_{[k]}\left|\widetilde{B}_{i}(k)\right|^{2}$. The scalings of the energy spectra by $k^{-5 / 3}$ and/or $k^{-7 / 3}$ have been examined in Ref. $[4,6]$. In both figures, we observe so-called 'pile-up' of energies at the tails $(k>16)$ of the spectra in Hall MHD (truncated) and MHD (truncated). The pile-up is obviously caused by the backscattering associated with the lack of wave-number space $k>k_{c}$. In LESes, the back-scattering is usually suppressed by a sub-grid-scale model (such as the eddy viscosity). In contrast to the pile-up at $k>16$, the spectra at low wavenumbers $(k \lesssim 10)$ are insensitive to truncation. The differences between the DNSes and the truncated runs of the Hall MHD and MHD, respectively, stay within about $10 \%$ at low wave-numbers.

The reason Hall MHD simulation was less affected by truncation than MHD turbulence simulation can be examined by studying the energy transfer functions. The energy transfer functions of the kinetic energy $E_{K}(k)$ and $E_{M}(k)$ can be described as

$$
\begin{aligned}
& T_{K}(k)=\sum_{[k]} \widetilde{V}_{i}(k)^{*} \times \\
& F\left[-\partial_{j}\left(V_{i} V_{j}-B_{i} B_{j}\right)-\partial_{i}\left(p+\frac{1}{2} B_{j} B_{j}\right)\right], \\
& T_{M}(k)=\sum_{[k]} \widetilde{B}_{i}(k)^{*} \widetilde{C}_{i}(k),
\end{aligned}
$$




$$
\widetilde{C}_{i}(k)=F\left[\epsilon_{i m n} \partial_{n}\left\{\epsilon_{n \alpha \beta}\left(V_{\alpha}-\epsilon j_{\alpha}\right) B_{\beta}\right\}\right],
$$

where $F[]$ denotes the Fourier transform. The symbol * denotes the complex conjugate. Figure 4 (a) shows $T_{K}(k)$ and $T_{M}(k)$ in Hall MHD (DNS) and Hall MHD (truncated), respectively. Both the DNS and the truncated run show that $T_{K}(k)<0$ for $k \lesssim 16$, and $T_{K}(k)>0$ for $k \gtrsim 16$. That is, forward energy transfer from large scales to small scales is dominant in the kinetic energy spectrum. The profile of $T_{M}(k)$ is somewhat different from that of $T_{K}(k)$. It can be divided into three regions; $k \simeq 1,2,3 \leq k<16$, and $k \geq$ 16. In the first region, $T_{M}(k)$ is positive, but its magnitude is not very large. In the second region, $T_{M}(k)$ is negative. The magnitude of $T_{M}(k)$ there is about one-third that of $T_{K}(k)$. In the third region, $T_{M}(k)$ is positive again.

Here we focus on the behavior of $T_{M}(k)$ at the boundary of the second and third regions, $k \simeq 16$, since the nature of the induction changes there. Figure 4 (b) shows $V(k):=\sqrt{E_{K}(k)}, J(k):=\sqrt{\sum_{[k]}\left|\widetilde{j_{i}}(k)\right|^{2}}$ and $(V(k)-\epsilon J(k))$. The two spectra, $V(k)$ and $J(k)$, have peaks at different wave-numbers. [From the energy spectra in Fig. 3, $V(k) \simeq$ $B(k)$ where $B(k):=\sqrt{E_{M}(k)}$, and we can roughly estimate $J(k) \sim k B(k) \sim k V(k)$, which gives a different peak position for $J(k)$.] The spectrum $V(k)-\epsilon J(k)$ changes its sign because of the differences in spectral profiles between $V(k)$ and $J(k)$, as $(V(k)-\epsilon J(k)) B(k)>0$ for $k \lesssim 16$, and $(V(k)-\epsilon J(k)) B(k)<0$ for $k \gtrsim 16$. We recall that $T_{M}(k)$ in Hall MHD (DNS) and Hall MHD (truncated) changes its signs at $k \simeq 16$. The magnetic energy transfer in Hall MHD turbulence is considered to change its direction either forward or backward at $k \simeq 16$, depending on the balance between $V(k)$ and $\epsilon J(k)$, or $\widetilde{\boldsymbol{V}}(k)$ and $\epsilon \widetilde{\boldsymbol{J}}(k)$ in 3D wavenumber space. It may be that the forward/backward transition, which is dominated by the balance between $V(k)$ and $\epsilon J(k)$, can be greater than the truncation effect, and that the magnetic energy transfer at $k \lesssim 16$ is not influenced much by truncation. We also note that, once a high $\epsilon$ is adopted, say $\epsilon=1, V(k)-\epsilon J(k)<0$ at all wave-numbers, so that the magnetic energy transfer profile should be totally modified. This remains to be simulated in the future.

\section{Concluding Remarks}

The effect of Fourier wave-number truncation is studied to obtain basic information for sub-grid-scale modeling of LESes. The numerical results for isotropic turbulence indicate that Hall MHD turbulence is more robust to truncation than MHD turbulence. In particular, the time evolution of the magnetic energy is not greatly affected by truncation even though the total current is considerably reduced by truncation. Energy transfer function analysis shows that the robustness of the magnetic field in Hall MHD turbulence can be attributed to the suppression of magnetic energy transfer by the Hall term. With these observations, we may be able to construct a sub-grid-scale
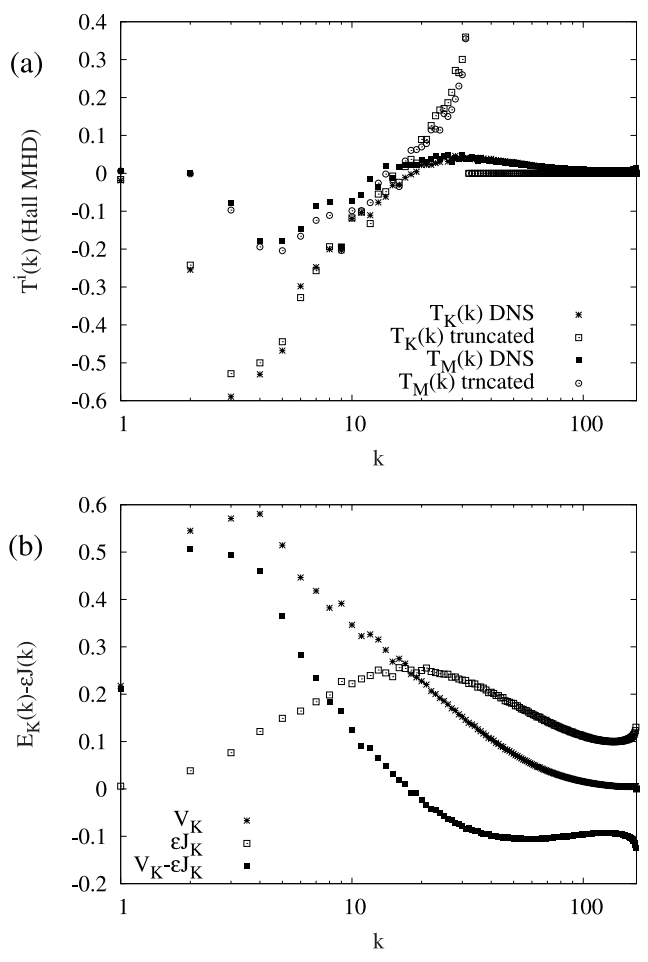

Fig. 4 Energy transfer functions $T_{K}(k)$ and $T_{M}(k)$ in Hall MHD (DNS) and Hall MHD (truncated).

model of the Hall term simply by reducing the magnetic energy transfer, so far as moderate value is given to Hall term. Though we have examined the Hall effect only for $\epsilon=0.05$, we need to vary $\epsilon$ over some typical values and see whether the conjecture is true or not. It is considered that an extreme case, $\epsilon=1$ for example, can show a totally different energy transfer mechanism. A sub-gridscale modeling for such an extreme case might be another target of our future works.

The numerical work was performed on the NEC SX-8 "LHD numerical analyses system", and on the HITACHI SR16000 "Plasma Simulator" of the National Institute for Fusion Science (NIFS), and supported by the NIFS Collaborative Research Program (NIFS09KNXN154, NIFS09KTAL018).

[1] H. Miura and N. Nakajima, Nucl. Fusion 50, 054006 (2010); H. Miura and N. Nakajima, in Fusion Energy 2008 (Proc. 22nd Int. Conf.Geneva, 2008) (Vienna: IAEA) CD-ROM file THP9/16 and http://wwwnaweb.iaea.org/napc/physics/FEC/FEC2008/html/index.htm.

[2] D. Biskamp, Magnetohydrodynamic Turbulence (Cambridge University Press, Cambridge, 2003).

[3] P. Mininni et al., J. Plasma Phys. 73, 377 (2007).

[4] D. Hori and H. Miura, Plasma Fusion Res. 3, S1053 (2008).

[5] H. Miura, submitted to J. Plasma Fusion Res. SERIES.

[6] H. Miura and D. Hori, J. Plasma Fusion Res. SERIES 8, 74 (2009). 